\title{
Anais do II Simpósio Brasileiro de Investigação de Doenças Neuromusculares
}

\section{Simpósio de Tecnologia Assistiva e Educação Especial da UNIFESP}

Data: 14 e 15 de maio de 2016

Local: Escola Paulista de Medicina, Universidade Federal de São Paulo (EPM/UNIFESP) 


\section{Prefácio}

As doenças neuromusculares envolvem diferentes afecções decorrentes do acometimento primário da unidade motora, sendo bem mais raras em crianças do que em adultos. Divididas topograficamente de maneira didática, estas evoluem para comprometimento motor e/ou respiratório, levando o paciente a incapacidades funcionais de atividades de vida diária, laborais e sociais.

O presente Simpósio objetivou apresentar os mais recentes trabalhos na área de Doenças Neuromusculares, bem como na área de Tecnologia Assistiva e Realidade Virtual no diagnóstico e tratamento destas doenças. Com o avanço contínuo e rápido desta era tecnológica, os pesquisadores têm buscado introduzir diferentes métodos para acelerar um diagnóstico, inovar na reabilitação e melhorar a qualidade de vida destes pacientes.

Saber avaliar as dificuldades, o momento certo da intervenção, a escolha do método é imprescindível para o profissional que lida diariamente com estas afecções, bem como é seu papel fundamental de atualizar das inovações da Tecnologia em Saúde.

Ft. Letícia Simões Ferreira

\section{Prêmio "JEAN-MARTIN CHARCOT"}

Serão premiados os três melhores trabalhos desenvolvido nos cursos de especialização em Intervenção fisioterapêutica nas Doenças Neuromusculares e Neurologia Clínica de 2015. 


\section{PROGRAMA}

14/05/2016 - sábado

Anf. Moacyr E. Alvaro

R.Botucatu, 862 - Vila Clementino

Sao Paulo - SP

8h

8h00 - Entrega do material na Secretaria

8h10 - Abertura do evento

Prof. Dr. Acary Souza Bulle Oliveira

Prof. Dra. Francis Meire Fávero

Prof. Dr. Ricardo M. Arida

8h20 - O uso de equipamentos para reabilitação de pacientes com doença de Parkinson

Prof. Ms. Carolina de Oliveira Souza - FMUSP

9h30 - Possibilidades de avaliação do controle motor em Distrofia Muscular

Prof. Dra. Cristina dos Santos Cardoso de Sá - UNIFESP

$10 \mathrm{~h} 10$ - COFFEE BREAK

10h30 - A importância do rastreador ocular na avaliação da criança com Atrofia Muscular Espinhal Tipo I

Prof. Dra. Mariana Callil Voos - USP

11h50 - Desenvolvimento de software para avaliação funcional:

experiência com FES-DMD-DATA

Prof. Dra. Fátima Caromano - USP

$12 \mathrm{~h} 30$ às $13 \mathrm{~h} 30$ almoço

13h30 - Pesquisa e desenvolvimento em design e tecnologia assistiva

Prof. Dr. Vagner Rogério dos Santos - UNIFESP

15h30 - COFFEE BREAK

15h50 - Realidade virtual na comunicação aumentativa e alternativa Prof. Ms. Tânia Brusque Crocetta - FMABC

16h30 - Premiação

17 h00 - Encerramento 
8h30 - Análise dos instrumentos de avaliação da dor em pacientes com Síndrome Pós-Poliomielite - revisão sistemática

Ft. Cleide Daiana Silva Miguel

Orientadora: Prof. Dra. Francis Meire Favero - UNIFESP

Convidado: Prof. Dr. Abrahão Augusto Juviniano Quadros UNIFESP

Presidente da Banca: Prof. Dr. Acary Souza Bulle Oliveira UNIFESP

9h50 - Relato de Experiência: Intervenção de fisioterapia respiratória em pacientes de Esclerose Lateral Amiotrófica Traqueostomizados

Ft. Marcelo Lima Moraes

Orientadora: Prof. Esp. Simone Gonçalves de Andrade Holsapfel - UNIFESP

Convidada: Prof. Ms. Thaís Massetti - USP

Presidente da Banca: Prof. Dr. Acary Souza Bulle Oliveira UNIFESP

10h30 - Efeitos do uso de órteses de membros inferiores na Distrofia Muscular de Duchenne - revisão da literatura

Ft. Fernanda Aparecida de Freitas Guimarães

Orientadora: Prof. Esp. Mariana de Abreu Rays - UNIFESP

Convidada: Prof. Dra. Cristina dos Santos Cardoso de Sá UNIFESP

Presidente da Banca: Prof. Dr. Acary Souza Bulle Oliveira UNIFESP

11h10 - Avaliação do controle de tronco e função de membro superior em pacientes com Distrofia Muscular de Duchenne

Ft. Ana Lúcia Yaeko da Silva Santos

Orientadora: Prof. Dra. Cristina dos Santos Cardoso de Sá UNIFESP

Convidada: Prof. Dr. Luis Fernando Grossklauss - UNIFESP Presidente da Banca: Prof. Dr. Acary Souza Bulle Oliveira UNIFESP

11 h50 - Relação entre a alteração motora e cognitiva em pacientes com Distrofia Muscular de Duchenne e em crianças saudáveis

Ft. Cristiany Munhoz De Lima Orientadora: Prof. Dra. Mariana Callil Voos - USP Convidado: Prof. Dr. Luis Fernando Grossklauss - UNIFESP Presidente da Banca: Prof. Dr. Acary Souza Bulle Oliveira UNIFESP

13h30 - Avaliação do desempenho motor na Esclerose Múltipla revisão sistemática 
Ft. Nádia Baggio Barreto Rodrigues

Orientadora: Prof. Dra. Francis Meire Favero - UNIFESP

Convidado: Prof. Dra. Michele Schult - USP

Presidente da Banca: Prof. Dr. Acary Souza Bulle Oliveira UNIFESP

14h10 - Comparação do tempo e dos movimentos compensatórios de marcha e do subir e descer degraus de meninos com Distrofia Muscular de Duchenne e meninos saudáveis

Ft. Rosana Hayasaka

Orientadora: Prof. Dra. Mariana Callil Voos - USP

Convidado: Prof. Dra. Fátima Aparecida Caromano - USP

Presidente da Banca: Prof. Dr. Acary Souza Bulle Oliveira UNIFESP

14h50 Caracterização do desempenho motor e funcional em portadoras sintomáticas com Distrofia Muscular de Duchenne: estudo de caso em série

Ft. Thiago Henrique da Silva Orientadora: Prof. Dra. Fátima Aparecida Caromano - USP Convidado: Prof. Dra. Mariana Callil Voos - USP Presidente da Banca: Prof. Dr. Acary Souza Bulle Oliveira UNIFESP

15h40 - Doenças genéticas diferentes com quadro clínico funcionais semelhantes: Miopatia de Bethlem e Distrofia Muscular Congênita de Ullrich - estudos de caso

Ft. Juliana Aparecida Rhein Telles Orientadora: Prof. Dra. Fátima Caromano - USP Convidado: Prof. Dra. Francis Meire Fávero - UNIFESP Presidente da Banca: Prof. Dr. Acary Souza Bulle Oliveira UNIFESP

16h20 - Correlação entre a funcionalidade e a força muscular de pacientes com diagnóstico de Esclerose Lateral Amiotrófica Ft. Karina Fontes Csibak Orientadora: Prof. Dra. Francis Meire Fávero - UNIFESP Convidado: Prof. Esp. Talita Dias da Silva - UNIFESP Presidente da Banca: Prof. Dr. Acary Souza Bulle Oliveira UNIFESP 


\section{O uso de equipamentos para reabilitação de pacientes com doença de Parkinson}

Carolina de Oliveira Souza

Fisioterapeuta da Faculdade de Medicina da Universidade de São Paulo

Parkinson's disease (PD) is neurodegenerative disorder characterized by resting tremor, rigidity, bradykinesia, and postural instability. Morris $(2000)^{1}$ described the theoretical framework supporting the use of PT in PD. Morris described task-specific strategies for improving the performance of activities. These strategies incorporate the use of external cues. External cues include visual, auditory, or proprioceptive stimuli that are either presented rhythmically. The use of portable cueing devices has proven effective for the rehabilitation of gait disorders in patients with PD. These devices are currently being developed, incorporating new technologies. Walking sticks ${ }^{2}$ and rolling walkers ${ }^{3}$ projecting a laser line on the or have shown efficacy in overcoming FOG and reducing falls in some but not all patients ${ }^{2}$. Light-emitting diodes (LEDs) ${ }^{4}$ and auditory devices incorporated into glasses are effective in improving gait parameters in laboratory settings ${ }^{5}$. The studies showed that even with the low serum level of levodopa, some patients could use visual and auditory cues to perform large stride lengths and increase gait velocity. Eyeglasses combining auditory-visual cueing are also effective on gait in advanced PD. Souza et al. ${ }^{6}$, used the device in 18 patients who received subthalamic nucleus DBS and gait improvement was observed in the cued condition. These devices hold great promise for becoming personalized, patient-tailored neurorehabilitation assistants in PD.

\section{References}

1. Morris ME. Movement disorders in people with Parkinson disease: a model for physical therapy. Phys Ther 2000;80:578-97. 
2.Donovan S, Lim C, Diaz N, Browner N, Rose P, Sudarsky LR. Laserlight cues for gait freez- ing in Parkinson's disease: an openlabel study. Parkinsonism Relat Disord 2011;17:240-5.

3.Bunting-Perry L, Spindler M, Robinson K, Noorigian MJ, Cianci $\mathrm{HJ}$, Duda JE. Laser light visual cueing for freezing of gait in Parkinson disease: a pilot study with male participants. J Rehabil Res Dev 2013; 50:223-30.

4.Ferrarin M, Brambilla M, Garavello L, Di Candia A, Pedotti A, Rabuffetti M. Microprocessor- controlled optical stimulating device to improve the gait of patients with Parkinson's disease. Med Biol Eng Comput 2004;42:328-32.

5.Lopez-Contreras WO, Higuera CA, Fonoff ET, Souza CO, Albicker U, Martinez JA. Listenmee and Listenmee smartphone application: synchronizing walking to rhythmic auditory cues to improve gait in Parkinson's disease. Hum Mov Sci 2014;37:147-56.

6.Souza CO, Voos MC, Chien HF, Brant R, Barbosa AF, Barbosa ER, et al. Combined auditory and visual cuing provided by eyeglasses influence gait performance in Parkinson disease patients submitted to DBS: a pilot study. Int Arch Med 2015;8:132-8.

\section{Possibilidades de avaliação do controle motor em Distrofia Muscular}

Cristina dos Santos Cardoso de Sá

Fisioterapeuta, Profa. Dra. Departamento Ciências do Movimento Humano, Curso de Fisioterapia, Universidade Federal de São Paulo.

As distrofias musculares (DM) são um grupo de desordens heterogêneas geneticamente determinadas, identificadas clinicamente por grande variabilidade do fenótipo e de gravidade e caracterizadas por fraqueza muscular. A maioria das DM compartilha a mesma característica patológica. Conforme o curso da doença, o tecido muscular sofre lesões que acarretam na formação de fibrose e 
posteriormente o tecido muscular é substituído por gordura. No entanto, o mecanismo de evolução e a velocidade em que ele ocorre em cada uma das formas específicas pode variar. A degeneração progressiva da musculatura esquelética acarreta na fraqueza e atrofia muscular, na instalação de contraturas musculares e deformidades tanto do esqueleto axial, do cíngulo escapular e dos membros superiores (MMSS), do cíngulo pélvico e dos membros inferiores (MMII) características inerentes de todo o grupo de DM. Sabe-se que o grau do envolvimento e do prejuízo destas musculaturas pode variar, e comprometer a funcionalidade desses indivíduos. Neste sentido, avaliar a funcionalidade dessa população é fundamental para a abordagem terapêutica. Essa avaliação fundamentada no controle motor permite entender a natureza e a causa da movimentação, a organização da movimentação e quais os mecanismos permitem essa organização. Portanto, entender o controle motor é fundamental para o sucesso da reabilitação dessa população visando às atividades de vida diária e tarefas motoras.

Para avaliar o controle motor é necessário identificar qual componente desse controle cada instrumento ou protocolo de avaliação deverá se utilizado. Uma vez que o controle motor depende de vários componentes: músculo esquelético, sinergias musculares, sistemas sensoriais, estratégias motoras, mecanismos compensatórios e adaptativos e representações internas. Dentre os sistemas de medição podemos destacar: cinemetria que se divide em análise cinemática e análise cinética do movimento, dinamometria e eletromigrafia. Essas análises requerem instrumentos específicos. Cada sistema de medição fornece informações sobre alguns componentes do controle motor. Em muitas situações é necessária a associação de diferentes instrumentos ou tipos de avaliação, por exemplo: cinemática que fornecerá informações sobre a velocidade, deslocamento, angulação, aceleração e tempo, e eletromiografia que fornecerá informações sobre a atividade muscular.

Palavras chaves: controle motor, fisioterapia, distrofia muscular 


\section{A importância do rastreador ocular na avaliação da criança com Atrofia Muscular Espinhal Tipo I}

Mariana Callil Voos

Professora Doutora da Universidade de São Paulo, Departamento de Fonoaudiologia, Fisioterapia e Terapia Ocupacional, São Paulo SP, Brasil.

A atrofia muscular espinhal (AME) é uma doença rara e neurodegenerativa, que afeta crianças e adultos. Gera atrofia muscular, prejudica os movimentos voluntários e, em muitos casos, dificulta a comunicação oral. Esta dissertação é composta por dois artigos. O Estudo 1 objetivou revisar a literatura sobre o desenvolvimento cognitivo de pessoas com AME. O Estudo 2 objetivou avaliar o desempenho visual, cognitivo e motor de crianças com AME tipo 1 (AME-I). O primeiro estudo foi uma revisão sistemática, com levantamento dos últimos 25 anos (de 1992 a 2017) nas bases de dados PUBMED/Medline, Web of Science e Scielo. Os descritores utilizados foram: spinal muscular atrophy e cognition. $O$ estudo 2 consistiu na avaliação de 24 crianças, divididas em dois grupos: 12 crianças com AME-I ( 3 a 9 anos, 9 meninos e 3 meninas) e 12 crianças saudáveis, pareadas por idade e sexo. As 24 crianças responderam a quatro tarefas de associação de figuras, com dificuldade crescente. As respostas foram detectadas por meio de um rastreador de olhar. Para o primeiro estudo foram localizados 43 artigos, desses, nove foram selecionados, de acordo com os critérios de inclusão e exclusão. O estudo apontou que o desempenho cognitivo de crianças com AME é heterogêneo. Três artigos indicaram que essas crianças têm desempenho cognitivo normal, três artigos indicaram que há atraso e outros três estudos relataram desempenho acima da média. De modo geral, as crianças com maior fraqueza muscular (AME-I) apresentaram maior comprometimento e estudos mais recentes detectaram esse atraso. O segundo estudo descreveu pior desempenho do grupo AME-I, em relação ao grupo controle 
(maior número de erros e maior tempo para execução das tarefas). Concluímos que, até o momento, poucos estudos investigaram aspectos cognitivos em crianças e adolescentes com AME. O desenvolvimento cognitivo de crianças com AME deve ser acompanhado, principalmente em crianças com AME-I. É necessário avaliar o desempenho cognitivo e, se necessário, propor intervenções para pessoas com AME. O desenvolvimento cognitivo adequado facilita a autonomia e interação. Os rastreadores de olhar podem estimular e aprimorar esse repertório.

\section{Escala de avaliação funcional da distrofia muscular de Duchenne (FES-DMD)}

Fátima Aparecida Caromano

Universidade de São Paulo, Departamento de Fonoaudiologia, Fisioterapia e Terapia Ocupacional, São Paulo SP, Brasil.

A Escala de Avaliação Funcional para distrofia muscular de Duchenne (FES-DMD) foi construída para descrever e quantificar os movimentos compensatórios desenvolvidos por esta população em atividades específicas. Tem quatro domínios: D1 - avalia o sentar e levantar da cadeira; D2-andar 10 metros; D3 - subir e descer escada; D4 - levantar e sentar no chão. A FES-DMD gera dados a partir de observação sistemática das atividades. A avaliação é realizada por terapeutas e envolve gravar e assistir os filmes de atividades funcionais, coleta de dados, geração de pontuações totais, medida de tempo de execução de atividades e geração de relatórios. Foram demonstradas sua confiabilidade intra e interexaminadores, assim como, estabelecido os períodos adequados para responsividade dos diferentes domínios da escala, com finalidade de que possa ser utilizada como biomarcador. Para facilitar todas essas tarefas, desenvolvemos um software denominado FES-DMD-DATA. Sua usabilidade foi demonstrada e seu registro efetuado. O uso de FES- 
DMD é simples, barato, prático e fornece registros permanentes e padronizados. A FES-DMD é sensível às mudanças funcionais ao longo do tempo, com capacidade de resposta consistente. Transforma o exame físico mais rápido e menos estressante para o paciente. Considerando todas as possibilidades de complicações clínicas, a FESDMD pode ser usada como complemento no acompanhamento da doença, até a fase de perda de deambulação, complementando a geração de informações para a adequada tomada de decisão clínica. Como a escala é ampla, estudos futuros devem desenvolver a versão simplificada.

Palavras-chaves: Muscular Dystrophy, evaluation, software, motor scale.

\section{Pesquisa e desenvolvimento em design e tecnologia assistiva}

Vagner Rogério dos Santos

Coordenador do grupo de pesquisa e desenvolvimento de pesquisa e design assistiva da UNIFESP

O design e a tecnologia são áreas de estudo com abordagens complementares. Sob a ótica do desenvolvimento de tecnologias assistivas, sua inter-relação garante que aspectos tecnológicos, antropométricos e de usabilidade sejam observados sem que ocorra o comprometimento estético da solução em tecnologia em desenvolvimento. Perceber que o usuário de tecnologia assistiva como um indivíduo, com percepções e estados emocionais. Portanto a pesquisa em design e tecnologia assistiva está à procura de soluções que combinem as relações de forma e função sem perder a compreensão que estados emocionais de pessoa com deficiência influenciam em seu uso. 


\section{Realidade virtual na comunicação aumentativa e alternativa}

Tânia Brusque Crocetta

Doutoranda pela Faculdade de Medicina do ABC

A interação da tecnologia, do jogo e da atividade motora pode auxiliar nas intervenções em indivíduos com deficiência motora, permitindo ao profissional de saúde avaliar o desempenho desses indivíduos com dados confiáveis. Portanto, o objetivo do nosso grupo é construir, validar e aplicar jogos virtuais e de realidade aumentada para aprendizagem motora, usando dispositivos físicos de contato (teclado e touch screen) e sensores de movimento (Kinect, Leap Motion e web cam). Os jogos oferecem medidas de desempenho e parâmetros de análise para auxiliar no planejamento de intervenções de profissionais de saúde. Outro objetivo é avaliar os efeitos de diferentes tipos de intervenções com estudos de aplicabilidade clínica, buscando evidências da eficácia deste tipo de tecnologia como forma de intervenção terapêutica em centros de reabilitação.

Palavras-chaves: realidade virtual, comunicação, reabilitação, avaliação, jogos

\section{Identificação de Instrumentos de Avaliação da Dor em} Pacientes com SPP- Revisão Sistemática

Cleide Daiana Silva Miguel1, Francis Meire Fávero $^{2}$

1 Especializanda do Curso de Especialização em Intervenção Fisioterapêutica nas Doenças Neuromusculares.

2 Professora Afiliada da Universidade Federal de São Paulo - Escola Paulista de Medicina

A dor é um dos principais sintomas da SPP, considerado o problema predominante interferindo na qualidade de vida do paciente. Objetivo: Identificar instrumentos de avaliação da dor de pacientes com síndrome pós- poliomielite através de uma revisão 
sistemática. Métodos: Foi realizado uma busca entre novembro de 2015 e janeiro de 2016, sem restrição de ano, no idioma inglês, nas seguintes bases de dados: MEDLINE/PUBMED, SCIELO e LILACS, sendo incluídos somente os artigos que apresentaram escalas de avaliação relacionadas com a dor de pacientes com SPP. Resultados: Foram encontradas 13 escalas de avaliação da dor aplicadas em pacientes com SPP. Conclusões: apesar dos instrumentos multidimensionais fornecerem dados mais amplos sobre dor, apresentam algumas limitações quanto à sua aplicação e, às vezes, tais instrumentos consistem em questionários muito longos e com um nível de complexidade tanto na aplicabilidade quanto na interpretação de seus resultados. Nesse sentido, nos ambientes de atendimento de dor aguda e de avaliações frequentes sem fins acadêmicos, a avaliação unidimensional da dor se faz a mais indicada.

Palavras-chave: Síndrome Pós-Poliomielite, Dor, Escalas, instrumentos, avaliação

Relato de Experiência: Intervenção de Fisioterapia Respiratória em Pacientes de Esclerose Lateral Amiotrófica Traqueostomizados

Marcelo Lima Moraes' ${ }^{1}$, Thaís Massetti2, Acary Souza Bulle Oliveira3, Simone Gonçalves de Andrade Holsapfel ${ }^{4}$

1 Especializando do Curso de Especialização em Intervenção Fisioterapêutica nas Doenças Neuromusculares.

2 Doutoranda da Universidade de São Paulo.

3. Professor da Universidade Federal de São Paulo - Escola Paulista de Medicina.

${ }^{4}$ Especialista da Universidade Federal de São Paulo - Escola Paulista de Medicina

A Esclerose Lateral Amiotrófica (ELA) é uma afecção degenerativa do sistema nervoso central (SNC), que envolve os 
neurônios motores do córtex cerebral, do tronco encefálico e no corno anterior da medula espinhal. A ELA se expressa clinicamente por meio de sinais de acometimento progressivo de neurônios motores superiores e inferiores, e a forma de início pode ser apendicular ou bulbar. O acometimento da musculatura respiratória ocorre em qualquer uma das formas e é a causa comum de morte. Objetivo. Relatar a experiência do tratamento de fisioterapia respiratória especializada após a traqueostomia em dois pacientes com ELA, S. D. 89 anos, sexo masculino, traqueostomizado à dois anos e oito meses e I.L.S. 74 anos, sexo feminino, traqueostomizada à dois anos e quatro meses. Método. Os pacientes foram atendidos no domicílio, em duas sessões diárias, com duração de uma hora cada. 0 tratamento realizado foi baseado em reexpansão pulmonar com o ambú e a remoção de secreções, com 0 aparelho de tosse mecanicamente assistida - TMA, com pressões pré-estabelecidas e mantidas diariamente. Resultados. Os pacientes tratados de forma especializada tem a possibilidade de índices reduzidos de internações hospitalares por infecções pulmonares. Conclusão. O tratamento respiratório especializado, reduz o número de ocorrências de infecções pulmonares e internações hospitalares.

Palavras-Chave: Esclerose Lateral Amiotrófica, musculatura respiratória, fisioterapia respiratória.

\section{Efeitos do Uso de Órteses de Membros Inferiores na Distrofia Muscular de Duchenne - Revisão Da Literatura}

Fernanda Aparecida de Freitas Guimarães ${ }^{1}$, Cristina dos Santos Cardoso de Sá2, Acary Souza Bulle Oliveira33, Mariana de Abreu Rays ${ }^{4}$ 1 Especializanda do Curso de Especialização em Intervenção Fisioterapêutica nas Doenças Neuromusculares.

2 Professora da Universidade Federal de São Paulo - Escola Paulista de Medicina. 
3 Professor da Universidade Federal de São Paulo - Escola Paulista de Medicina.

${ }^{4}$ Mestranda da Universidade Federal de São Paulo - Escola Paulista de Medicina.

A Distrofia Muscular de Duchenne é uma doença genética na qual ocorre uma degeneração progressiva do tecido muscular de rápida evolução. Programas de reabilitação associados ao uso de órtese têm sido uma proposta que busca lentificar as perdas motoras e aumentar a independência. Objetivo. Verificar os efeitos do uso das órteses de membros inferiores em pacientes com Distrofia Muscular de Duchenne. Método. Realizou-se um levantamento bibliográfico de artigos científicos publicados no período de 1991 a 2015; utilizou-se as bases de dados Medline/Pubmed, Lilacs, Scielo e PEDro. Resultados. Selecionou-se um total de 10 artigos científicos sobre uso de órteses e sua representação na reabilitação, funcionalidade e marcha do paciente com DMD. Conclusão: As órteses para membros inferiores podem apresentar efeitos benéficos em relação ao uso. Quando associado ao alongamento muscular e posicionamento do complexo articular irá prevenir as deformidades, favorecer ao prolongamento e melhoria da funcionalidade da marcha.

Palavras-chave: Distrofia Muscular de Duchenne; Aparelhos Ortopédicos; Reabilitação.

$2^{\circ}$ Prêmio "JEAN-MARTIN CHARCOT "

Avaliação do Controle de Tronco e Função de Membro Superior em Pacientes com Distrofia Muscular de Duchenne

Ana Lúcia Yaeko da Silva Santosi; Francis Meire Favero²; Luis Fernando Grossklauss3; Acary Souza Bulle Oliveira 3; Mariana Cunha Artilheiro ${ }^{2}$; Mariana Callil Voos ${ }^{4}$; Cristina dos Santos Cardoso Sá ${ }^{\text {. }}$

1 Especializanda do Curso de Especialização em Intervenção Fisioterapêutica nas Doenças Neuromusculares. 
2 Professora Afiliada da Universidade Federal de São Paulo - Escola Paulista de Medicina

3 Universidade Federal de São Paulo, Departamento de Neurologia/Neurocirurgia, São Paulo SP, Brasil;

4 Universidade de São Paulo, Departamento de Fonoaudiologia, Fisioterapia e Terapia Ocupacional, São Paulo SP, Brasil;

5 Universidade Federal de São Paulo, Departamento Ciências do Movimento Humano, São Paulo SP, Brasil.

O objetivo foi avaliar o controle de tronco e o desempenho funcional dos membros superiores (MMSS), e relacionar o nível de controle de tronco, a funcionalidade MMSS e o nível funcional de paciente com distrofia muscular de Duchenne (DMD). Estudo transversal composto por 35 pacientes diagnosticados com DMD, média de 13,26 anos, submetidos a avaliação do controle de tronco pela Segmental Assessment of Trunk Control (SATCo), o desempenho de MMSS pelo teste Jebsen Taylor (TJT) e o nível funcional pela escala Vignos. O controle de tronco total foi mais prevalente dentre os pacientes, no subteste da escrita do TJT os pacientes tiveram período de tempo prolongado para executá-lo, o nível funcional predominante indicou que eram pacientes cadeirantes, os quais sentavam ereto e eram capazes de conduzir a cadeira e realizar atividades de vida diária na cama ou na cadeira. Houve correlação entre controle de tronco, funcionalidade de MMSS e nível funcional, e a mesma foi fraca. O estudo caracteriza o controle de tronco e a funcionalidade de MMSS de pacientes com DMD, como também demonstra a relação entre eles e com o nível funcional.

Palavras-chave: Distrofia Muscular de Duchenne; Avaliação; Fisioterapia. 


\section{Relação entre a Alteração Motora e Cognitiva em Pacientes com Distrofia Muscular de Duchenne e em Crianças Saudáveis}

Cristiany Munhoz De Lima1; Francis Meire Favero ${ }^{2}$; Luis Fernando Grossklauss ${ }^{3}$; Acary Souza Bulle Oliveira ${ }^{3}$; Fátima Aparecida Caromano ${ }^{4}$; Mariana Callil Voos ${ }^{4}$.

1 Especializanda do Curso de Especialização em Intervenção Fisioterapêutica nas Doenças Neuromusculares.

2 Professora Afiliada da Universidade Federal de São Paulo - Escola Paulista de Medicina

3 Universidade Federal de São Paulo, Departamento de Neurologia/Neurocirurgia, São Paulo SP, Brasil;

4 Universidade de São Paulo, Departamento de Fonoaudiologia, Fisioterapia e Terapia Ocupacional, São Paulo SP, Brasil;

Introdução: Os pacientes com distrofia muscular de Duchenne (DMD ) mostram comprometimento motor e também pode ter disfunção cognitiva, tais como dificuldades de habilidades visuoespaciais, linguagem, memória de trabalho e processos fonológicos. Objetivo: O objetivo deste estudo será: descrever as alterações motoras e cognitivas de um grupo de pacientes com DMD e de crianças saudáveis, investigar possíveis relações entre o desempenho cognitivo e motor tanto no grupo com DMD quanto no grupo controle. Método: Estudo prospectivo observacional transveral, será aplicados testes que avaliam as habilidades cognitivas e motoras dos participantes com idade de 8-21 anos. Será utilizado as escalas MFM e Vignos, trabalhando teste de memória será utilizado Mini- Exame do Estado Mental, teste de fluência verbal (animais), ordem dos dígitos (ordem direta e reversa) e o teste do relógio. Resultados: Foram observadas correlações fortes entre as variáveis cognitivas (MEEM vs. Teste do relógio; MEEM vs. dígitos ordem inversa, teste do relógio vs. MEEM, Teste do relógio vs. ordem inversa, dígitos ordem inversa vs. MEEM, dígitos ordem inversa vs. Teste do relógio) 
Conclusão: Podemos concluir que não houveram correlações significativas entre as variáveis cognitivas e motoras.

Palavras-chave: distrofia muscular de Duchenne, cognição , atividade motora, habilidades motoras, desenvolvimento psicomotor

\section{Análise de Instrumentos da Avaliação Motora na Esclerose Múltipla - Revisão Sistemática}

Nádia Baggio Barreto Rodrigues ${ }^{1}$; Michele Schult ${ }^{2}$; Acary Souza Bulle Oliveira ${ }^{3}$; Francis Meire Favero ${ }^{4}$

1 Especializanda do Curso de Especialização em Intervenção Fisioterapêutica nas Doenças Neuromusculares.

2 Universidade de São Paulo, Brasil.

3 Universidade Federal de São Paulo, Departamento de Neurologia/Neurocirurgia, São Paulo SP, Brasil.

${ }^{4}$ Professora Afiliada da Universidade Federal de São Paulo - Escola Paulista de Medicina, Brasil.

Introdução: A Esclerose Múltipla (EM), doença auto-imune desmielinizante, é a principal causadora de deficiência neurológica não traumática em adultos jovens, causando disfunções sensoriais, cognitivas e motoras. Devido a sua complexidade, é necessário utilizar escalas de avaliação padronizadas, as quais promovem maior direcionamento da avaliação e tratamento. Objetivo: Identificar os instrumentos de avaliação motora utilizados em pacientes com Esclerose Múltipla - revisão sistemática. Método: Foi realizada busca eletrônica nas bases de dados LILACS e Medline/PubMED utilizando os descritores "Multiple Sclerosis", "Motor Skills" e "Disability Evaluation". Resultados: Foram analisados 11 artigos selecionados de acordo com critérios de inclusão e exclusão, verificando-se considerável quantidade de avaliações no total e por artigo, sendo que todas as escalas funcionais validadas para a população brasileira foram também validadas para a população de EM. Conclusão: A 
utilização de avaliações selecionadas criteriosamente promove avaliação fidedigna da funcionalidade dos pacientes, bem como a verificação da evolução da doença.

Palavras-chave: Esclerose Múltipla, Escalas, Avaliação, Motora, Análise

10 Prêmio "JEAN-MARTIN CHARCOT "

Comparação do Tempo e dos Movimentos Compensatórios da Marcha e do Subir e Descer Degraus em Meninos com Distrofia Muscular de Duchenne e Meninos Saudáveis

Rosana Hayasaka1; Acary Souza Bulle Oliveira² ; Fátima Aparecida Caromano ${ }^{3}$; Mariana Callil Voos ${ }^{3}$.

1 Especializanda do Curso de Especialização em Intervenção Fisioterapêutica nas Doenças Neuromusculares.

2 Universidade Federal de São Paulo, Departamento de Neurologia/Neurocirurgia, São Paulo SP, Brasil;

3 Universidade de São Paulo, Departamento de Fonoaudiologia, Fisioterapia e Terapia Ocupacional, São Paulo SP, Brasil;

Introdução: A distrofia muscular de Duchenne (DMD) é caracterizada pela fraqueza muscular progressiva, simétrica, bilateral, com início em músculos proximais de membros inferiores. Crianças com DMD desenvolvem movimentos compensatórios para andar, subir e descer escadas. Para avaliar esses movimentos a Functional Evaluation Scale for Duchenne Muscular Dystrophy (FES-DMD) é utilizada. Objetivos: (1) Comparar o tempo e os movimentos compensatórios durante a marcha e o subir e descer degraus em meninos com DMD e meninos saudáveis e (2) comparar o tempo e os movimentos compensatórios dessas três atividades (andar, subir, descer degraus). Método: A amostra foi composta por filmes de 32 meninos com DMD (média de idade: 10,0 anos) e de 38 meninos saudáveis (média de idade de 9,2 anos. Os movimentos 
compensatórios foram analisados utilizando os domínios marcha e subir e descer degraus da FES-DMD e o tempo, cronometrado em segundos. Resultados: Ao compararmos os grupos controle e DMD, não houve diferença no tempo de marcha, porém houve diferença entre os tempos das atividades de subir e descer degraus. Os meninos com DMD apresentaram maior número de movimentos compensatórios em todas as atividades funcionais analisadas, principalmente durante a marcha. Conclusão: Os movimentos compensatórios dos meninos com DMD deram eficiência à marcha, pois o tempo nessa atividade foi semelhante ao do grupo controle. 0 mesmo não ocorreu ao subir e descer escadas. Apesar do aumento no número de movimentos compensatórios, o tempo necessário para essas atividades foi maior do que no grupo controle.

Palavras-chave: distrofia muscular de Duchenne; avaliação; atividade motora; função executiva; locomoção.

Caracterização do Desempenho Motor e Funcional de Portadoras Sintomáticas de Distrofia Muscular de Duchenne: Série de Casos

Thiago Henrique da Silva ${ }^{1}$, Isabela Pessa Anequini2, Francis Meire Fávero3, Mariana Callil Voos ${ }^{4}$, Acary Souza Bulle Oliveira ${ }^{5}$, Carlos Bandeira de Mello Monteiro ${ }^{4}$, Fatima Aparecida Caromano ${ }^{4}$

1 Especializando do Curso de Especialização em Intervenção Fisioterapêutica nas Doenças Neuromusculares.

2 Mestranda da Universidade de São Paulo

3 Professora Afiliada da Universidade Federal de São Paulo - Escola Paulista de Medicina.

4 Universidade de São Paulo, Departamento de Fonoaudiologia, Fisioterapia e Terapia Ocupacional, São Paulo SP, Brasil;

5 Universidade Federal de São Paulo, Departamento de Neurologia/Neurocirurgia, São Paulo SP, Brasil; 
A distrofia muscular de Duchenne (DMD) acomete indivíduos do sexo masculino. Porém, mulheres também são afetadas em casos raros. Aproximadamente $8 \%$ das portadoras de DMD têm sintomas musculares ou cardíacos. A identificação precoce de deficiências funcionais e motoras em portadoras de DMD pode direcionar a tomada de decisão clínica. Estudos epidemiológicos e clínicos com portadoras de DMD podem esclarecer esta questão. Objetivo: Investigar as alterações motoras e funcionais de nove pacientes do sexo feminino com distrofinopatia diagnosticada por estudos clínicos, patológicos e genéticos. Método: Trata-se de uma série de casos retrospectivos, descrevendo nove portadoras do sexo feminino DMD, que apresentaram sintomas clínicos. Os dados foram coletados a partir avaliação de força muscular (MRC) e desempenho funcional (FES-DMD). Os pacientes foram primeiramente diagnosticados por biópsia muscular com imunohistoquímica (distrofina). Depois disso, foram especificadas as alterações genéticas (MLPA). Resultados: A idade de início dos sintomas apresentados variou (01-50 anos de idade). As deleções foram encontradas em sete pacientes. Pseudo hipertrofia de panturrilhas, movimentos compensatórios e aumento progressivo no tempo de execução de tarefas funcionais (sentar/levantar da cadeira e do chão, andar e subir/descer escadas) foram observados em sete casos. A fraqueza muscular foi detectada em todos os casos. Sete pacientes apresentaram fraqueza muscular assimétrica. As pacientes mais jovens mostraram comprometimento menos grave do que os mais velhos. Conclusão: Fraqueza muscular progressiva e comprometimento funcional podem ser observados em portadoras DMD. Portanto, as funções musculares e as tarefas funcionais devem ser precocemente avaliadas nesta população.

Palavras-chave: Distrofia muscular de Duchenne, atividade motora, Distrofinopatia, portadora feminina 
Genótipos Diferentes com Fenótipos Semelhantes: Miopatia de Bethlem e Distrofia Muscular Congênita de Ullrich - Estudo de Casos

Juliana Aparecida Rhein Telles ${ }^{1}$; Isabela Pessa Anequini2; Francis Meire Fávero3; Mariana Callil Voos ${ }^{4}$; Acary Souza Bulle Oliveira ${ }^{5}$, Fatima Aparecida Caromano 4

1 Especializando do Curso de Especialização em Intervenção Fisioterapêutica nas Doenças Neuromusculares.

2 Mestranda da Universidade de São Paulo

3 Professora Afiliada da Universidade Federal de São Paulo - Escola Paulista de Medicina.

4 Universidade de São Paulo, Departamento de Fonoaudiologia, Fisioterapia e Terapia Ocupacional, São Paulo SP, Brasil;

5 Universidade Federal de São Paulo, Departamento de Neurologia/Neurocirurgia, São Paulo SP, Brasil;

A Miopatia de Bethlem (MB) e a Distrofia Muscular Congênita de Ullrich (DMCU) são consequências de mutação na matriz extracelular da proteína colágena VI. São raras e ainda não descritas sua funcionalidade. Objetivo: Descrever a funcionalidade. Resultados: O paciente com miopatia de Bethlem é mulher de 20 anos, filha de pais saudáveis, com desenvolvimento neuromotor normal até doze meses. Primeiros sintomas com um ano de idade. Aos doze anos apresentava levantar miopático. Realizou quatro procedimentos cirúrgicos, sendo o primeiro no tendão de calcâneo. Aos 18 anos apresentava capacidade vital forçada entre 45 e 50\%. Sintomas dermatológicos presentes: hiperqueratose folicular e tendência à formação de aderência em cicatrizes. Grupos musculares mais afetados: flexores e extensores de membros inferiores, dorsiflexores e flexores plantares bilateralmente. Paciente com distrofia muscular congênita de Ullrich, 12 anos sempre sofreu muitas quedas e logo começou a andar na ponta dos pés. Aos 5 anos só deambulava com apoio de antepé, levantava do chão somente com auxílio de cadeira 
com muita dificuldade, apresentava amiotrofia difusa em membros inferiores e membros superiores proximais e distais, hiperlordose lombar, frouxidão ligamentar e ausência de contraturas. Alguns grupos musculares sofreram redução de força mais severa, sendo os mais distais de membros superiores, correspondentes aos flexores e extensores de cotovelo, flexores e extensores de punho. A contratura mais intensa observada foi de flexão de joelhos. Conclusão: Foram pesquisadas força muscular, contraturas e testes funcionais e descritos déficits motores graves nos dois indivíduos, sendo que 0 caso MB apresentou mais brando.

Palavras-chave: Distrofia muscular, atividade motora, avaliação, escala

30 Prêmio "JEAN-MARTIN CHARCOT "

Correlação Entre a Funcionalidade e a Força Muscular de Pacientes com Diagnóstico de Esclerose Lateral Amiotrófica

Karina Fontes Csibak1; Mariana Callil Voos ${ }^{2}$; Acary Souza Bulle Oliveira ${ }^{3}$; Fatima Aparecida Caromano ${ }^{2}$; Francis Meire Favero ${ }^{4}$

1 Especializando do Curso de Especialização em Intervenção Fisioterapêutica nas Doenças Neuromusculares.

2 Universidade de São Paulo, Departamento de Fonoaudiologia, Fisioterapia e Terapia Ocupacional, São Paulo SP, Brasil;

3 Universidade Federal de São Paulo, Departamento de Neurologia/Neurocirurgia, São Paulo SP, Brasil;

${ }^{4}$ Professora Afiliada da Universidade Federal de São Paulo - Escola Paulista de Medicina.

Introdução: A Esclerose Lateral Amiotrófica (ELA) é uma doença neurodegenerativa causada pela lesão em neurônios motores superiores e inferiores. Um dos principais sintomas que envolve essa doença é a perda da força muscular (FM), ocasionando a dependência funcional. A escala da Medida da Função Motora (MFM) demonstrou 
boa confiabilidade e homogeneidade na sua aplicação, permitindo o uso desse instrumento para medir a função motora nas doenças neuromusculares, esse instrumento pode apresentar sensibilidade para analisar as alterações funcionais em um intervalo de tempo menor entre as avaliações, sendo este resultado necessário para as mudanças terapêuticas de acordo com a evolução da doença.

Objetivo: Analisar a correlação entre a funcionalidade e a força muscular de pacientes com diagnóstico de ELA. Método: Foram incluídos 12 pacientes com ELA que apresentavam a marcha selecionados no Setor de Investigação de Doenças Neuromusculares da Universidade Federal de São Paulo/Escola Paulista de Medicina, submetidos Mini Exame do Estado Mental afim de verificar o grau de compreensão, escala de Vignos, correlacionar escalas funcionais e FM como Amyotrophic Lateral Sclerosis Functional Rating Scale - Revised (ALSFRS-R), a MFM - Dimensões e a FM (MRC\%), para análise foi utilizado testes de correlação de Pearson (dados paramétricos) e/ou Spearman (dados não-paramétricos), considerando coeficientes fortes de $(r>0,70)$ ou moderados $(0,40<r>0,70)$. Resultados: Correlações entre as escalas funcionais e a força muscular (MRC\%), a FM Proximal de MMSS versus ALSFRS ( $r=0,73 ; p=0,007)$, FM Distal de MMSS versus ALSFRS $(r=0,74, p=0,005)$, FM Proximal de MMII versus MFM-D1 $(r=0,77, p=0,003)$, FM Distal de MMII versus MFMD1 $(r=0,80, p=0,002)$, FM Proximal de MMSS versus MFM-D3 $(r=0,76, p=0,004)$ e FM Distal de MMSS versus MFM-D3 ( $r=0,64$, $p=0,022)$. Conclusão: Existe forte correlação entre as escalas funcionais ALSFRS e dimensões 1 da MFM com a força muscular dos segmentos distais e proximais de MMSS e MMII, esse dado também foi apresentado na correlação entre a dimensão 3 da MFM e a FM Proximal de MMSS, porém entre a Dimensão 3 da MFM com a FM distal de MMSS a correlação foi classificada como moderada. Esses dados representam claramente que a MFM pode ser uma escala mais sensível para avaliar os pacientes com ELA, sabendo que o segmento 
distal de MMSS é o mais comprometido na fase inicial e que a evolução é de distal para proximal nesta doença.

Palavras-chave: Esclerose Lateral Amiotrófica, Força Muscular, Função, Escalas e avaliação. 